\title{
Degradation-Reduction Features of Electrophysical Characteristics of Irradiated Gallium Phosphide Light-Emitting Diodes
}

\author{
R.M. VernyduB ${ }^{a}$, O.I. KYrylenko ${ }^{a, *}$, O.V. Konoreva ${ }^{b}$, \\ O.I. RADKEVYCH ${ }^{c}$, D.P. STRATILAT ${ }^{d}$ AND V.P. TARTACHNYK ${ }^{d}$ \\ ${ }^{a}$ National Pedagogical Dragomanov University, Kyiv, Ukraine \\ ${ }^{b}$ E.O. Paton Electric Welding Institute of the NAS of Ukraine, Kyiv, Ukraine \\ "SE "SRI of Microdevices" STC "Institute for Single Crystals" of the NAS of Ukraine, \\ Kyiv, Ukraine \\ ${ }^{d}$ Institute for Nuclear Research of the NAS of Ukraine, Kyiv, Ukraine
}

Received: 28.04.2021 \& Accepted: 14.06.2021

Doi: 10.12693/APhysPolA.140.141 *e-mail: etfa@ukr.net

\begin{abstract}
The results of studies of the current-voltage and electroluminescent characteristics of the original GaP LEDs and those irradiated by electrons with $E=2 \mathrm{MeV}$ and $F=8.2 \times 10^{16} \mathrm{~cm}^{-2}$ are presented. The data of isochronous annealing of samples containing defects of technological and radiation origin are analyzed. Radiation damage coefficients for the lifetime of minority charge carriers in diodes irradiated by electrons and $\alpha^{++}$particles with $E=28 \mathrm{MeV}$ have been determined.
\end{abstract}

topics: LED, radiation, annealing, dislocation

\section{Introduction}

The spread of powerful information arrays based on the use of modulated streams of charged particles-electrons in the leading cable or electromagnetic waves in the open air space is accompanied by the occurrence of inevitable heat losses and dissipation of the emitter's energy; this also complicates the possibility of further increasing the frequency density in one channel.

The use of optical vibrations in fiber-optic communication lines significantly increases the information capacity of the transmitting-receiving path, increases the recording capacity, does not create electromagnetic interference, and does not emit information, thereby ensuring the secrecy of communication, and does not require additional shielding systems from external fields.

Typical modern sources of light signals in the optoelectronic region are semiconductor lasers and LEDs, whose stability of the characteristics and the absence of failures during the period of use depend on the reliability of operation of the entire transmitting-receiving complex [1].

The development of the space industry of microelectronics, nuclear power, and controls for accelerating devices expands the field of application of optoelectronic converters. At the same time, it becomes necessary to study the nature of defects of radiation origin, thermal stability, degradation, and restoration of the main characteristics in them, as well as to search for opportunities to predict radiation resistance and develop methods for increasing it. In recent years, there has been an intensive development of both traditional and newest methods of obtaining, researching, and using GaP crystals, which are basic for the production of simple, reliable, and cheap LEDs on their basis [2-6].

The results presented below relate mainly to studies of degradation-reduction processes revealed as a result of the analysis of the spectral and current-voltage characteristics (CVC) of GaP LEDs irradiated by electrons with $E=2 \mathrm{MeV}$. Taking into account the breadth of the field of the modern practical application of LEDs, the authors also deemed it necessary to consider the features of the operational characteristics of emitters of various spectral ranges and to determine the constants of their radiation resistance.

\section{Experiment}

We have investigated commercial epitaxial GaP LEDs, whose $n$-regions were doped with $\mathrm{Te}=2 \times 10^{17} \mathrm{~cm}^{-3} \quad\left(E_{a}^{\mathrm{Te}}=0.8 \mathrm{eV}\right) ; \quad$ and $p$-regions - with $\mathrm{Zn}$ and $\mathrm{O}$ - simultaneously $\left(E_{a}^{\mathrm{Zn}}=0.064 \mathrm{eV} ; E_{a}^{\mathrm{O}}=0.90 \mathrm{eV} ; \mathrm{Zn}\right.$ is an acceptor, $\mathrm{O}$ is a donor). The $n$-GaP substrate was grown by the Czochralski method with a carrier concentration of $(5-7) \times 10^{17} \mathrm{~cm}^{-3}$. The sample size was $1 \mathrm{~mm}^{2}$. 
Electroluminescence spectra were obtained using a BLK-C F1000-VIS NIR-1 spectrometer (StellarNet Inc) for the range of $190-850 \mathrm{~nm}$ in the temperature range $77-400 \mathrm{~K}$.

The CVC was measured in the temperature range of 77-300 K by an automated complex in the modes of a current generator and a voltage generator.

Irradiation by electrons with $E=2 \mathrm{MeV}$ was carried out in a pulsed mode on an IPP-6 accelerator with an electron beam current $I=4 \mathrm{~mA}$ at room temperature, the glass lens of the diode was preliminarily removed. The U-120 cyclotron served as a source of $\alpha^{++}$particles with $E=28 \mathrm{MeV}$.

Isochronous annealing of irradiated samples was performed in the temperature range $T=20-500^{\circ} \mathrm{C}$ with a period $t=20 \mathrm{~min}$.

\section{Results and discussion}

The emission spectra of the green diode $\mathrm{GaP}(\mathrm{N})$ obtained at various excitation currents are shown in Fig. 1a.

The short-wavelength doublet band N-2TA is the result of phonon repetitions of the line of the exciton $\mathrm{N}$ bound to the isoelectronic impurity of nitrogen; the broad maximum $h \nu=2.15 \mathrm{eV}$ is also a phonon replica, but an exciton trapped by a nitrogen pair $\mathrm{NN}_{1}$ with a minimum distance between atoms $[7,8]$.
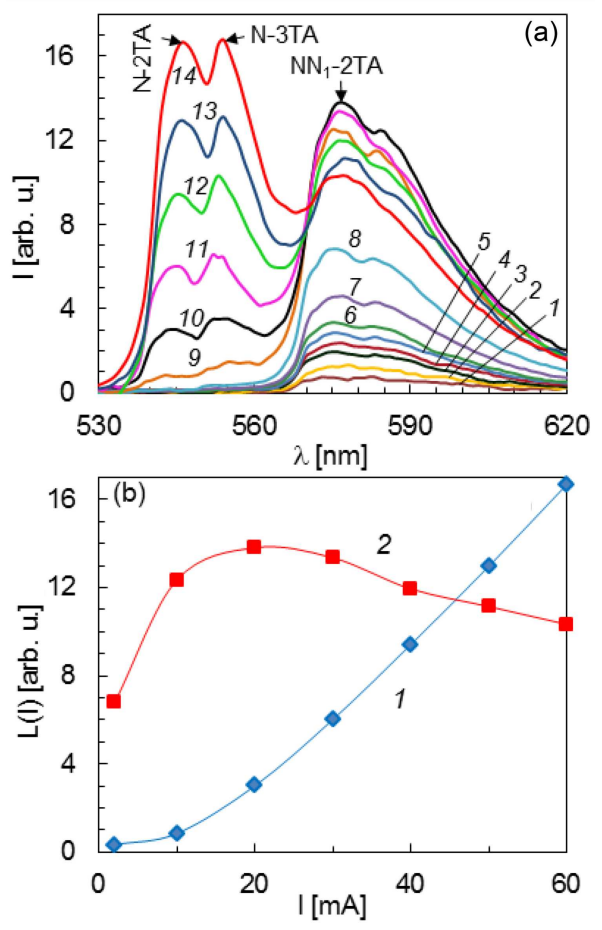

Fig. 1. (a) The emission spectra of a green GaP LED, measured at various excitation currents $(77 \mathrm{~K}): 1-0.05,2-0.2,3-0.4,4-0.6$, $5-0.8,6-1,7-1.5,8-2,9-10,10-$ $20,11-30,12-40,13-50,14-60 \mathrm{~mA}$. (b) Dependence of the luminescence intensity of the $\mathrm{N}-2 \mathrm{TA}(1)$ and $\mathrm{NN}_{1}-2 \mathrm{TA}$ (2) bands on the excitation current.

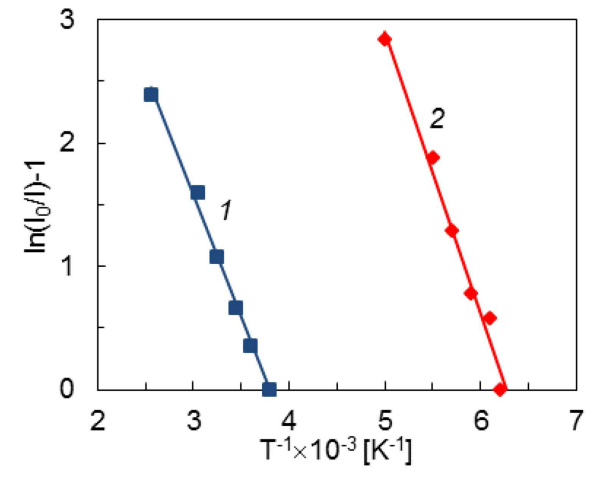

Fig. 2. Temperature dependence of the radiation intensity of the $\mathrm{NN}_{1}$ (1) and N-2TA (2) bands of the original $\mathrm{GaP}(\mathrm{N}) \mathrm{LED}$.

Figure 1b shows the dependence of the glow intensity of both bands on the injection level of minority charge carriers in the range of operating currents $(I=2-60 \mathrm{~mA})$; it can be seen that $L(I)$ is linear only for $h \nu=2.27 \mathrm{eV}$, which should be taken into account when matching the optical characteristics of the source-receiver in fiber-optic communication lines.

The temperature dependence of the intensity of their radiation is shown in Fig. 2.

The activation energy for luminescence quenching can be estimated using the Mott formula [9] for the quantum yield of luminescence

$$
\eta=\frac{1}{1+q \exp \frac{E_{A}}{k_{\mathrm{B}} T}},
$$

where $q$ is the constant and $k_{\mathrm{B}}$ is the Boltzmann constant.

The numerical values of the activation energy for quenching of two bands are $E_{A 1}=0.19 \mathrm{eV}$ (for $h \nu=2.15 \mathrm{eV}$ ) and $E_{A 2}=0.17 \mathrm{eV}$ (for $h \nu=2.27 \mathrm{eV})$. The insignificant distance between $E_{A 1}$ and $E_{A 2}$ may indicate the probable genetic relationship of band 2, namely, with exciton bound on an isolated $\mathrm{N}$ atom, and not on the $\mathrm{NN}_{1}$ pair [8]. Both values are greater than the thermal dissociation energy of the bound exciton, which is not higher than $40 \mathrm{MeV}$ [8]. Therefore, it is obvious that at the thermal quenching of the bands, defects also take place with a much greater depth of the levels. By fixing the current value on the family of CVC recorded at different temperatures, it is possible to reproduce the curve of isochronous annealing of the diode.

Figure 3 shows the dependence of the voltage drop of the original $\mathrm{GaP}(\mathrm{Zn}, \mathrm{O}) \mathrm{LED}$ and that irradiated by electrons with $E=2 \mathrm{MeV}$, $F=8.2 \times 10^{16} \mathrm{~cm}^{-2}$ on the annealing temperature, taken at a constant current.

The change in $U(T)$ of the original diode in general terms can be considered a two-stage process with temperature intervals of $50-180^{\circ} \mathrm{C}$ and $180-490^{\circ} \mathrm{C}$. In the irradiated diode, recovery occurs within 3 stages: $20-50^{\circ} \mathrm{C}, 50-120^{\circ} \mathrm{C}, 120-270^{\circ} \mathrm{C}$, 


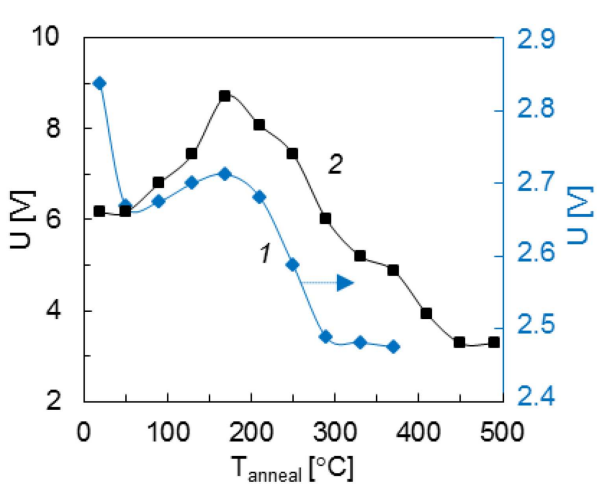

Fig. 3. Dependence of the voltage at the original (1) and electron-irradiated $(2, E=2 \mathrm{MeV}$, $\left.F=8.2 \times 10^{16} \mathrm{~cm}^{-2}\right) \mathrm{GaP}(\mathrm{Zn}, \mathrm{O})$ diode on the annealing temperature, measured at $T=300 \mathrm{~K}$. The annealing period was $20 \mathrm{~min}$.

and the second stage is negative - an increase in temperature leads to a partial return of the sample to a state with a higher voltage drop across it.

The difficulty in interpreting the mechanism of annealing an object with a $p-n$ junction lies in the existence of two regions with different types of conductivity.

In GaP single crystals, as is known from [10], the nature of the annealing of the $n$ - and $p$-material is different. Samples of $n$-type irradiated by electrons with $E=1-2 \mathrm{MeV}$ are characterized by the main stage $120-180^{\circ} \mathrm{C}$ at which the phosphorus vacancies $\mathrm{V}_{\mathrm{P}}$ come into motion; the main annealing of $p$-type single crystals is observed in the range of $250-300^{\circ} \mathrm{C}$ where $\mathrm{V}_{\mathrm{Ga}}$ is activated.

In the irradiated diode, in the temperature range of $20-50^{\circ} \mathrm{C}$, a sharp drop in voltage $\Delta U=3-8 \mathrm{~V}$ is observed, followed by which it rises again at the next stage. It should be noted here that in a homogeneous irradiated crystal, regardless of the type of conductivity, this stage does not manifest itself. So, it can be hoped that its existence is due to the presence in the diode of an interface of various types of conductivity with an increased density of dislocations, where point defects with low activation energy generated by irradiation can accumulate in the vicinity of the Cottrell atmosphere.

The negative stage of $50-120^{\circ} \mathrm{C}$ is absent in irradiated homogeneous crystals. This feature may indicate the formation of complexes of radiation defects with impurities, which decay even at $T>150^{\circ} \mathrm{C}$.

The last stage of annealing at $120-370^{\circ} \mathrm{C}$ is the result of the superposition of the stages of annealing $\mathrm{V}_{\mathrm{P}}$ and $\mathrm{V}_{\mathrm{Ga}}$, which, due to their high concentration in the irradiated sample, cease to separate.

The main function of LEDs is radiative, and therefore their radiation resistance is determined primarily by changes in the intensity of the glow.

It is known that in gallium phosphide and in a solid solution based on its GaAsP, nonradiative

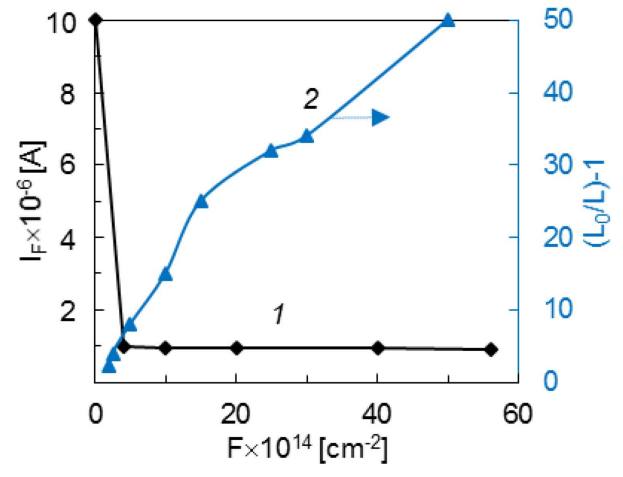

Fig. 4. Dose dependence of the luminescence intensity of the $\mathrm{GaP}(\mathrm{Zn}, \mathrm{O})$ LED irradiated by electrons with $E=2 \mathrm{MeV}$ (1) and function $\frac{L_{0}}{L}-1$ (2) measured at $T=300 \mathrm{~K}$.

levels of radiation defects decrease the lifetime of minority charge carriers $[11,12]$. The effectiveness of their action is estimated by the value of the damage coefficient of the lifetime $K_{\tau}$ of minority charge carriers. Within doses, where the difference between the reciprocal lifetime of the irradiated and original diodes is proportional to the fluency

$$
\begin{aligned}
& \frac{1}{\tau_{F}}-\frac{1}{\tau_{0}}=K_{\tau} F, \\
& \frac{\tau_{0}}{\tau_{F}}=1+\tau_{0} K_{\tau} F .
\end{aligned}
$$

But, according to [12]:

$$
\frac{\tau_{0}}{\tau_{F}}=\frac{L_{0}}{L},
$$

then

$$
\frac{L_{0}}{L}-1=\tau_{0} K_{\tau} F
$$

The value of $K_{\tau}$ is determined from the slope of the dose dependence of the function $\frac{L_{0}}{L}-1$.

Figure 4 shows the dose dependence of the luminescence intensity of the GaP LED, as well as the function $\left(\frac{L_{0}}{L}-1\right)$. Damage coefficients $K_{\tau 1}=$ $0.71 \times 10^{-8} \mathrm{~cm}^{2} \mathrm{~s}^{-1}$ and $K_{\tau 2}=1.67 \times 10^{-7} \mathrm{~cm}^{2} \mathrm{~s}^{-1}$ can be distinguished on both curves. In the case of irradiation with electrons with $E=1-2 \mathrm{MeV}$, the primary introduced defects are point structure disturbances and create a decisive influence on the lifetime of minority charge carriers.

For comparison, we present the data obtained by us after irradiation of the LED GaP with heavy charged particles $\left(\alpha^{++}, 28 \mathrm{MeV}, F=10^{12} \mathrm{~cm}^{-2}\right)$, when disordering regions are introduced simultaneously with point violations. In this case, $K_{\tau}=3 \times 10^{-5} \mathrm{~cm}^{2} \mathrm{~s}^{-1}$ which is orders of magnitude higher than the values obtained by us and given above.

\section{Conclusions}

The depth of occurrence of the levels causing quenching of the main electroluminescence bands was estimated $\left(E_{A 1}=0.19 \mathrm{eV}, E_{A 2}=0.17 \mathrm{eV}\right)$. 
An analysis of the annealing process showed that in unirradiated samples of the first stage of annealing at $50-180^{\circ} \mathrm{C}$ it is possible to compare the migration of phosphorus vacancies to the sinks; the second stage of annealing at $180-490^{\circ} \mathrm{C}$ can be compared with the diffusion of $\mathrm{V}_{\mathrm{Ga}}$.

The irradiated sample is characterized by the presence of three stages: $20-50{ }^{\circ} \mathrm{C}, 50-120^{\circ} \mathrm{C}$, and $120-270^{\circ} \mathrm{C}$. The first of it can be associated with the annealing of point defects concentrated near dislocation piles within the boundaries of the $p$ - and $n$-region interface; the last, main stage is the result of the superposition of the stages of annealing $V_{P}$ and $\mathrm{V}_{\mathrm{Ga}}$, caused by their high concentration.

\section{References}

[1] V.I. Karas, L.A. Nazarenko, I.V. Karas, LEDs: Physics, Manufacturing Technology, Application, Kharkiv, Ukraine 2012.

[2] S. Das, S. Chaudhuri, K. Mandal, ECS J. Solid State Sci. Technol. 5, 3059 (2016).

[3] S. Assali, I. Zardo, S. Plissard, M.A. Verheijen, J.E.M. Haverkort, E.P.A.M. Bakkers, in: Proc. Int. Conf. on Indium Phosphide and Related Materials (IPRM), 2013, p. 1.
[4] A. Martin, S. Combrié, A. Rossi, G. Beaudoin, I. Sagnes, F. Raineri, Photon. Res. 6, 43 (2018).

[5] J.H. Kim, T. Kawazoe, M. Ohtsu, Adv. Opt. Technol. 2015, 236014 (2015).

[6] M. Darnon, R. Varache, M. Descazeaux, T. Quinci, M. Martin, T. Baron, D. Munoz, AIP Conf. Proc. 1679, 040003 (2015).

[7] Zh. Pankov, Optical Processes in Semiconductors, Moscow 1973.

[8] A.A. Bergh, P.J. Dean, Light-Emitting Diodes, Oxford 1976.

[9] V.V. Antonov-Romanovskij, Kinetics of Photoluminescence of Crystals, Moscow 1966.

[10] E.Yu. Brailovskii, I.D. Konozenko, V.G. Makarenko, V.S. Manzhara, V.P. Tartachnik, in: Proc. Int. Conf. on Lattice Defects in Semiconductors, Vol. 6, 1974 , p. 8.

[11] O.V. Konoreva, M.V. Lytovchenko, Ye.V. Malyj, M.B. Pinkovska, V.V. Shlapatska, Semicond. Phys. Quant. Electron. Optoelectron. 18, 312 (2015).

[12] B.H. Rose, C.E. Barnes, J. Appl. Phys. 53, 1772 (1982). 